تاريخ القبول

$r \cdot 1 r / 1 Y / 1 V$

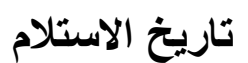

$r+1 r / .9 / r \varepsilon$

\title{
The Study Of $8_{1}^{+}$Isomeric State For
} ${ }_{28}^{70} \mathrm{Ni},{ }_{30}^{72} \mathrm{Zn},{ }_{32}^{74} \mathrm{Ge},{ }_{34}^{76} \mathrm{Se},{ }_{36}^{78} \mathrm{Kr}$ and $\left.{ }_{38}^{80} \mathrm{Sr} r\right)$ Even-Even Isotones

\section{With $\mathbf{N}=42$}

\section{Imad M.Ahmed Zena H. Salih Mutasim M.Yousif University of Mosul College of Education / Department of Physics}

\section{Abstract}

In this paper we study of the characteristics of the $8_{1}^{+}$isomeric state of $\left({ }_{28}^{70} \mathrm{Ni},{ }_{30}^{72} \mathrm{Zn},{ }_{22}^{74} \mathrm{Ge},{ }_{34}^{76} \mathrm{Se},{ }_{36}^{78} \mathrm{Kr}\right.$ and $\left.{ }_{38}^{80} \mathrm{Sr}\right)$ isotones with $(N=42)$ and the reduced electric quadrupole transition probability in unit of Weisskopf and calculate these values $\uparrow$ and $\downarrow$ in unit of $\left(e^{2} b^{2}\right)$, depending on these values we calculate the electric quadrupole moment $Q_{0}$ and the deformation parameter $\beta$, the single particle deformation parameter $\beta_{s p}$ and the mean deformation parameter $\delta$ are also calculated. The square of the emitted photon energy $\left(\hbar^{2} w^{2}\right)$ and the moment of inertia $\left(2 \vartheta / \hbar^{2}\right)$ for these states are also calculated.

الخلاصة

تم دراسة شصائص الحالات الايزوميرية ( ذات $\quad{ }_{38}^{80} \mathrm{Sr},{ }_{36}^{78} \mathrm{Kr},{ }_{34}^{76} \mathrm{Se},{ }_{32}^{74} \mathrm{Ge},{ }_{30}^{72} \mathrm{Zn},{ }_{28}^{70} \mathrm{Ni}$ )

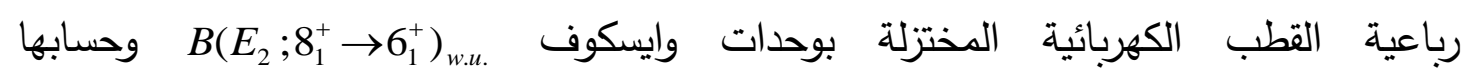
بوحدات 


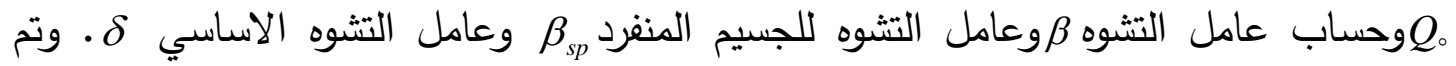

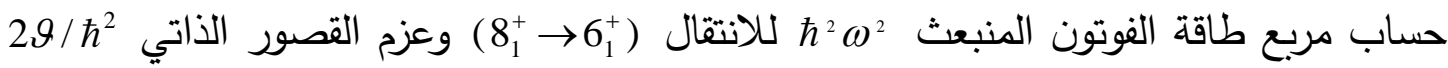

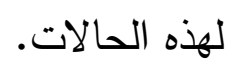

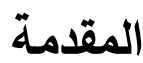

تعد دراسة الحالات الايزوميرية مهمة في التعرف على خصائص بعض النوى بشكل خاص في النوى غير المستقرة في منطقة النوى الثقيلة جدا، وتكون الحالة الارضية اكثر استقرارا (مستقرة في الغالب) من حالات التهيج. وتعد دراسة الانتقالات في النوى غير المستقرة ذات التهات

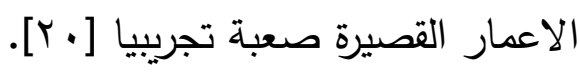
تم الحصول على معلومات في التحول الحاصل في المدار 2P $2 P_{3 / 2}$ للحالة الارضية لنواة

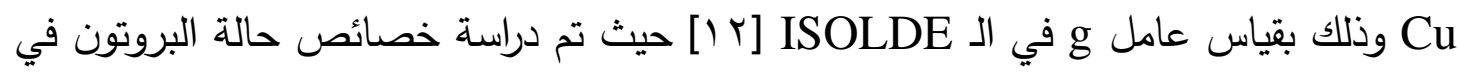
المدار f/2 • ان دراسة احتمالية الانتقال المختزلة لهذه النواة تبين السلوك الجماعي للحالات الواطئة لغاية N=46 [9]. تم دراسة نظائر Cu بالاعتماد على نموذج الجسيم المنفرد لغاية

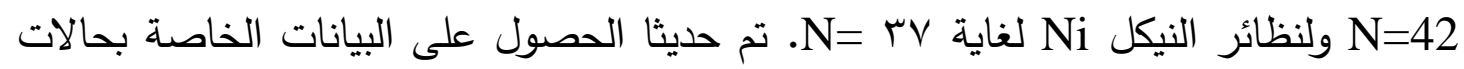
الجسيم المنفرد لنواة الـ NSOLDE وصف الحالات بازدواج الجسيمات في قلب الـ

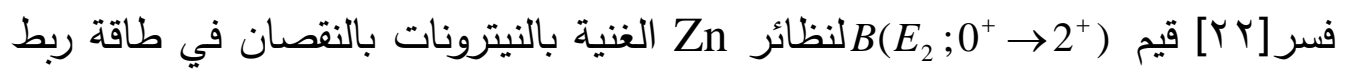

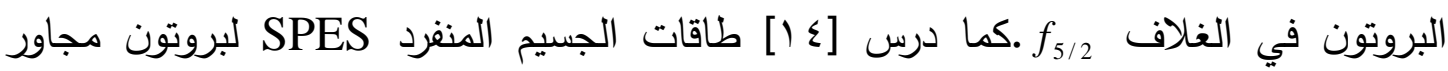
ونيترون مجاور لنواة الـ لـ في المنطقة إ) دراسة ملئ الاغلفة فوق الغلاف 28 الى المدار و/2 و، ودلت الدراسة على طبيعة المهتز

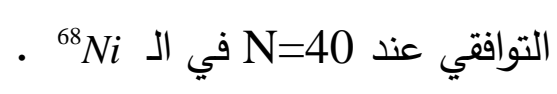
قدّم [1] دراسة للحالات الايزوميرية + $8_{1}^{+}$ الزوجية-الزوجية، وقدم دراسة للحالات الايزوميرية +8 للنوى من No42.

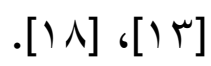

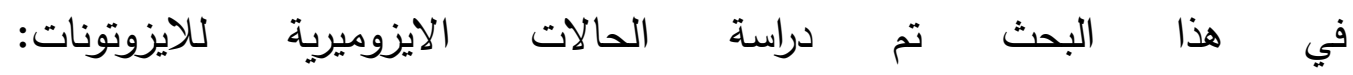
، وذلك للتعرف على القيم التجريبية لـ

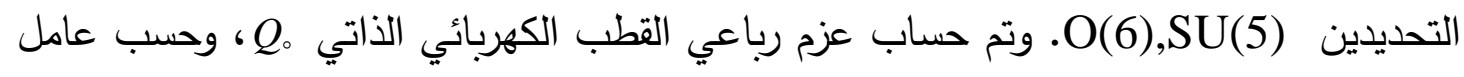

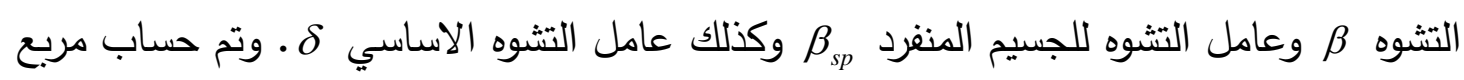


طاقة الفوتون المنبعث الحالات الايزوميرية.

النظريـة :- (النية

تم حساب احتمالية الانتقال رباعية القطب الكهربائية بوحدات ق قيمة هذه الاحتمالية

بوحدات وايسكوف للجسيم المنفرد وباستخدام المعادلة [Mark, 2003]:

$B\left(E_{2}\right)_{e^{2} b^{2}}=B\left(E_{2}\right)_{w . u} * 5.943 \times 10^{-6} A^{4 / 3}$

تم استخدام نموذج البوزونات المتفاعلة IBM-1 لغرض حساب احتمالية الانتقال رباعية

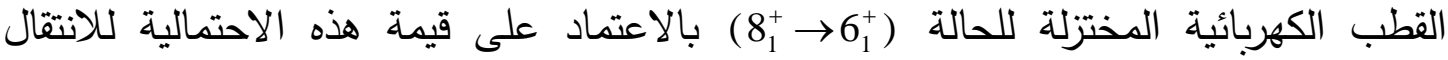
( وذلك لعدم توفر هذه القيمة عمليا) باستخدام المعادلتين الخاصتين بالتحديدين الاهتزازي وكاما الناعمة لغرض حساب ( $B\left(E_{2} ; I+2 \rightarrow I\right)=\alpha_{2}^{2} \frac{1}{4}(L+2)(2 N-L)$

$B\left(E_{2} ; I+2 \rightarrow I\right)=\alpha_{2}^{2} \frac{(I+2)}{2(I+5)} \frac{1}{4}(2 N-I)(2 N+I+8)$

تعطى العلاقة بين $B\left(E_{2}, J_{i} \rightarrow J_{f}\right) \downarrow=B\left(E_{2}, J_{i} \rightarrow J_{f}\right) \uparrow \times \frac{\left(2 J_{f}+1\right)}{\left(2 J_{i}+1\right)}$

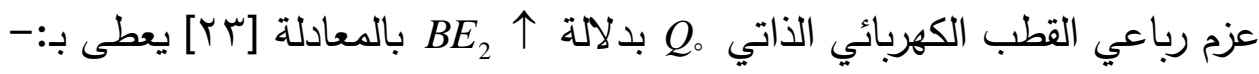

$e^{2} Q_{0}^{2}(J \rightarrow J-2)=B\left(E_{2}\right) \times \frac{32 \pi}{15} \frac{(2 J-1)}{(J-1)} \frac{2(J-1)}{J}$

ويمكن حساب عزم رباعي القطب الكهربائي (eb)(J) بدلالة عزم رباعي القطب الكهربائي الذاتي هQ للحالة ( $Q(J)(b)=\frac{J(2 J-1)}{(J+1)(2 J+3)} Q_{0}(b)$

عامل التشوه $\beta$ بدلالة $\left(B E_{2}\right)$ من المعادلة [8] يعطى بـ:$\beta=\left[B\left(E_{2}\right) \uparrow\right]^{4 / 2}\left[3 Z R_{0}^{2} / 4 \pi\right]^{-1}$

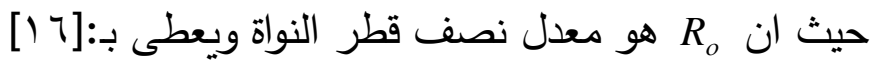
$R_{0}^{2}=0.0144 A^{2 / 3} b$ 
اما عامل التشوه للجسيم المنفرد $\beta_{s p}$ فيعطى بالمعادلة [lV]

$\beta_{2 s p}=1.59 / Z$

حيث ان Z ال هو العدد الذري.

بينما عامل التشوه الاساس $\delta$ ــ [11]

$\delta=0.895 \beta^{2}$

ان مربع طاقة الفوتون المنبعث

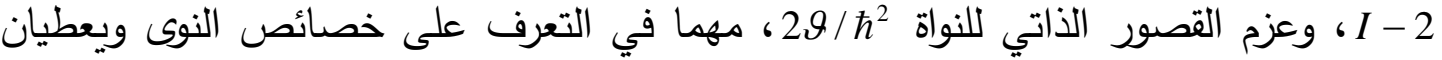

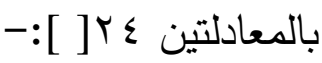

$\hbar^{2} \omega^{2}=\left(J^{2}-J+1\right)\left[\frac{E(J \rightarrow J-2)}{2 J-2}\right]^{2}$

$\frac{2 \vartheta}{\hbar^{2}}=\frac{4 J-2}{E(J \rightarrow J-2)}$

حيث ان Eيمثل فرق الطاقة بين المستويين E الحسابات والنتائج

تم استخدام المعادلة (1) في حساب احتمالية الانتقال رباعية القطب الكهربائية المختزلة بوحدات باستخدام المعادلة (ع) والجدول (1) يوضح ذلاك.

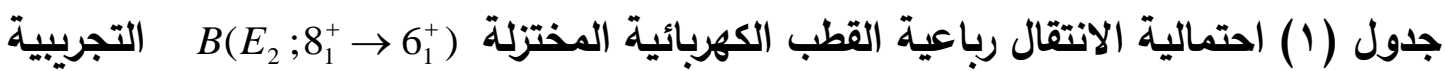
بوحدات w.u

\begin{tabular}{|c|c|c|c|}
\hline Nuclei & $B\left(E_{2} ; 8_{1}^{+} \rightarrow 6_{1}^{+}\right)_{\text {w.u. }}$ & $B\left(E_{2} ; 8_{1}^{+} \rightarrow 6_{1}^{+}\right)_{e^{2} b^{2}} \downarrow$ & $B\left(E_{2} ; 8_{1}^{+} \rightarrow 6_{1}^{+}\right)_{e^{2} b^{2}} \uparrow$ \\
\hline${ }_{28}^{70} \mathrm{Ni}_{42}$ & $0.655^{a}$ & $\ldots 11$ & $\cdots 10$ \\
\hline${ }_{30}^{72} Z n_{42}$ & $30.4^{b}$ & $\ldots 0 \leqslant$ & $\cdots v \cdot 7$ \\
\hline${ }_{32}^{74} G e_{42}$ & $39^{c}$ & $\cdots v_{1}$ & $\ldots .917$ \\
\hline${ }_{34}^{76} \mathrm{Se}_{42}$ & $82^{d}$ & .0107 & $\cdot r \cdot \varepsilon$ \\
\hline${ }_{36}^{78} \mathrm{Kr}_{42}$ & $85^{e}$ &. .171 &.$r 19$ \\
\hline${ }_{38}^{80} \mathrm{Sr}_{42}$ & $137^{f}$ &.$r \wedge$. & ( \\
\hline
\end{tabular}

$\mathrm{a}=[21]$

$\mathrm{b}=\left(\alpha_{2}^{2}=3.8\right)$ using equ (2) 
$\mathrm{c}=$ using equ $(3)\left(\alpha_{2}^{2}=2.75\right)$

$\mathrm{d}=[6]$

$\mathrm{e}=[3]$

$\mathrm{f}=[5]$

وباستخدام المعادلتين (0) و (T) تم حساب عزم رباعي القطب الكهربائي الذاتي $Q_{0}$ و و للانتقال (6 $\left.6_{1}^{+} 8_{1}^{+}\right)$للنوى قيد الدراسة والجدول (r) يوضح ذلك.

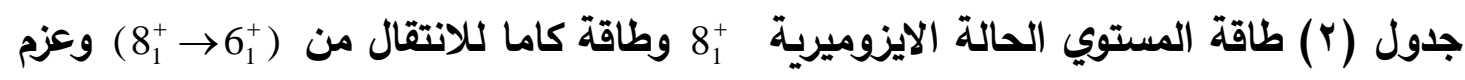

رباعي القطب الكهربائي م و

\begin{tabular}{|c|c|c|c|c|c|}
\hline Nuclei & $E 8_{1}^{+}(\mathrm{keV})$ & $E 2_{1}^{+}(k e V)$ & $E_{\gamma}\left(8_{1}^{+} \rightarrow 6_{1}^{+}\right)(\mathrm{keV})$ & $Q_{0}(e b)$ & $Q_{J}(e b)$ \\
\hline${ }_{28}^{70} N i_{42}$ & $2860^{a}$ & $1259^{a}$ & IN1 & $. .19 . r$ & צחזו.. \\
\hline${ }_{30}^{72} \mathrm{Zn}_{42}$ & $3569.7^{b}$ & $652^{b}$ & $917 . r$ & I.MTI & $.9 \mathrm{rV}$ \\
\hline${ }_{32}^{74} G e_{42}$ & $3681^{c}$ & $595^{c}$ & 1111.7 & $1.0 \mathrm{rT}$ & $1 . .79$ \\
\hline${ }_{34}^{76} \mathrm{Se}_{42}$ & $3269.8^{d}$ & $559^{d}$ & $1 \cdot V \cdot r$ & $r . r \leq q$ & $1.0 \mathrm{VA}$ \\
\hline${ }_{36}^{78} \mathrm{Kr}_{42}$ & $2993.5^{e}$ & $455^{e}$ & $1.10 . \mathrm{V}$ & r.r. & ס 1.7 \\
\hline${ }_{38}^{80} \mathrm{Sr}_{42}$ & $2700.42^{f}$ & $385^{f}$ & $q r V$ & r... & Y.111 \\
\hline
\end{tabular}

$a=[21]$

$\mathrm{b}=[19]$

$\mathrm{c}=[4]$

$\mathrm{d}=[6]$

$\mathrm{e}=[3]$

$\mathrm{f}=[5]$

استخدمت قيم

عامل التشوه للجسيم المنفرد ع $_{s p}$ باستخدام المعادلة (9) وعامل التشوه الاساس $\delta$ باستخدام المعادلة ( • () وعرضت هذه القيم في الجدول (ب). بينما استخدمت المعادلة (1 ( ) لحساب مربع طاقة الفوتون المنبعث

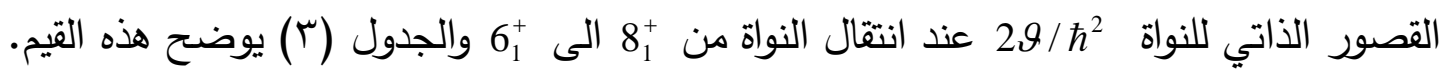




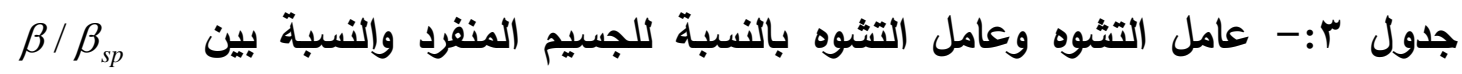
وعامل التثوه الاساس ومربع الطاقة الدورانية وعزم رباعي القطب الكهبائي

\begin{tabular}{|c|c|c|c|c|c|c|}
\hline Nuclei & $\beta$ & $\beta_{s p}$ & $\beta / \beta_{s p}$ & $\delta$ & $\hbar^{2} \omega^{2}\left(M e V^{2}\right)$ & $2 \vartheta / \hbar^{2}(M e V)^{-1}$ \\
\hline${ }_{28}^{70} \mathrm{Ni}_{42}$ & ...rT & .0 .077 & $. . \leqslant 1 Y \leqslant$ & $\ldots \ldots \leqslant q$ & ...9vr & 174.94 \\
\hline${ }_{30}^{72} Z n_{42}$ & $. .1 \leqslant 19$ &. .04 & r.A.q &. .191 & $. r \leq \leqslant$ & TY.V \\
\hline${ }_{32}^{74} \mathrm{Ge}_{42}$ & .101 & $\ldots$. $\leqslant १ \vee$ & r.lAr & 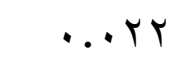 &. .509 & r..99 \\
\hline${ }_{34}^{76} \mathrm{Se}_{42}$ & $\because r) 0$ & $\cdots \leqslant 7$ & $\{.71 \leqslant$ & $\cdots \leqslant 1$ &. .990. & r9.1 \\
\hline${ }_{36}^{78} \mathrm{Kr}_{42}$ & $\because r \cdot V$ & $\cdots \leq \varepsilon$ & $5.79 V$ & ..r人 & . & r9.0 \\
\hline${ }_{38}^{80} S_{r_{42}}$ & $\because r \leq q$ & $\cdots \leq 1$ & $0.97 \pi$ & .000 & r.roor & r...IV \\
\hline
\end{tabular}

تم رسم العلاقة بين طاقة الحالة الايزوميرية +8 كدالة للعدد الذري Z وكما موضح في

(1) (1) (1) (1)

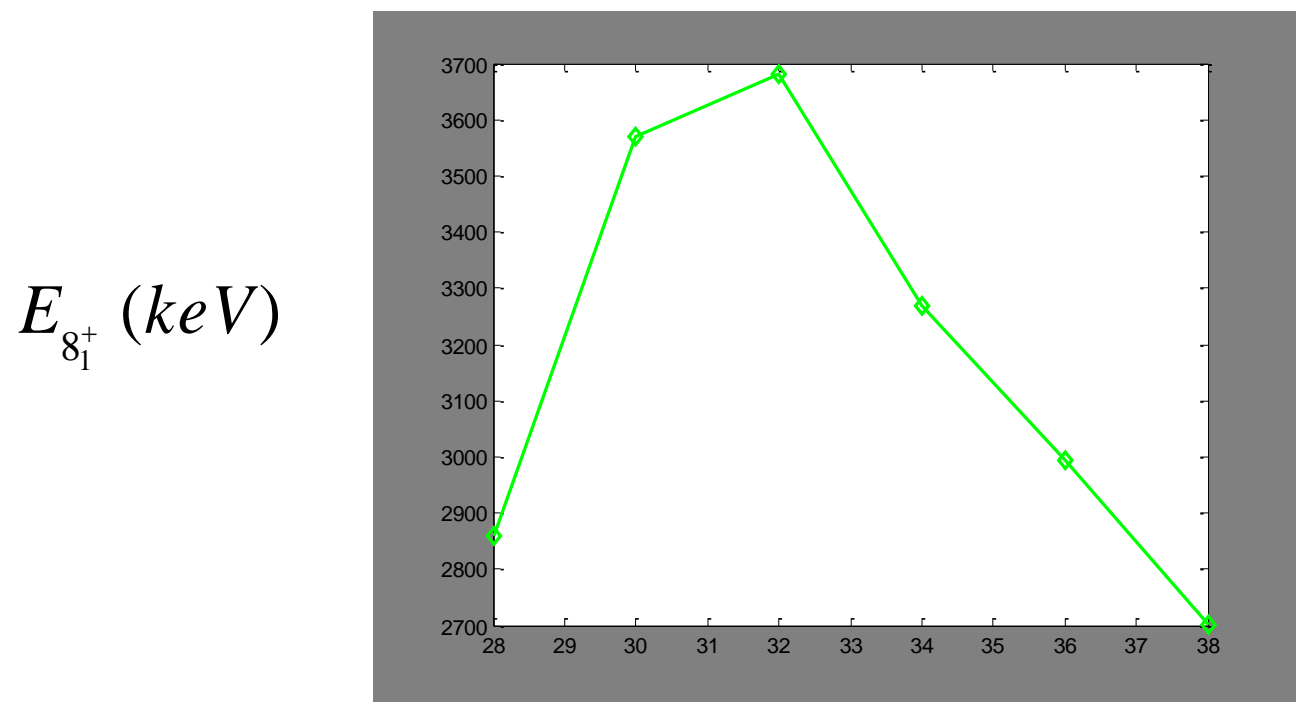

Atomic Number $(Z)$

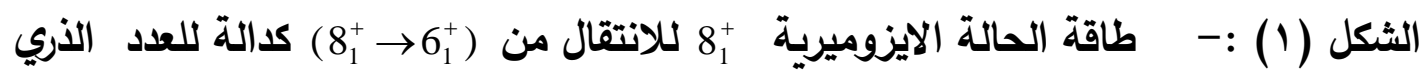
ل للايزوتونات

كما تم رسم العلاقة بين احتمالية الانتقال رباعية القطب الكهربائية المختزلة

B $B\left(E_{2} ; 8_{1}^{+} \rightarrow 6_{1}^{+}\right)_{e^{2} b^{2}} \downarrow$ 


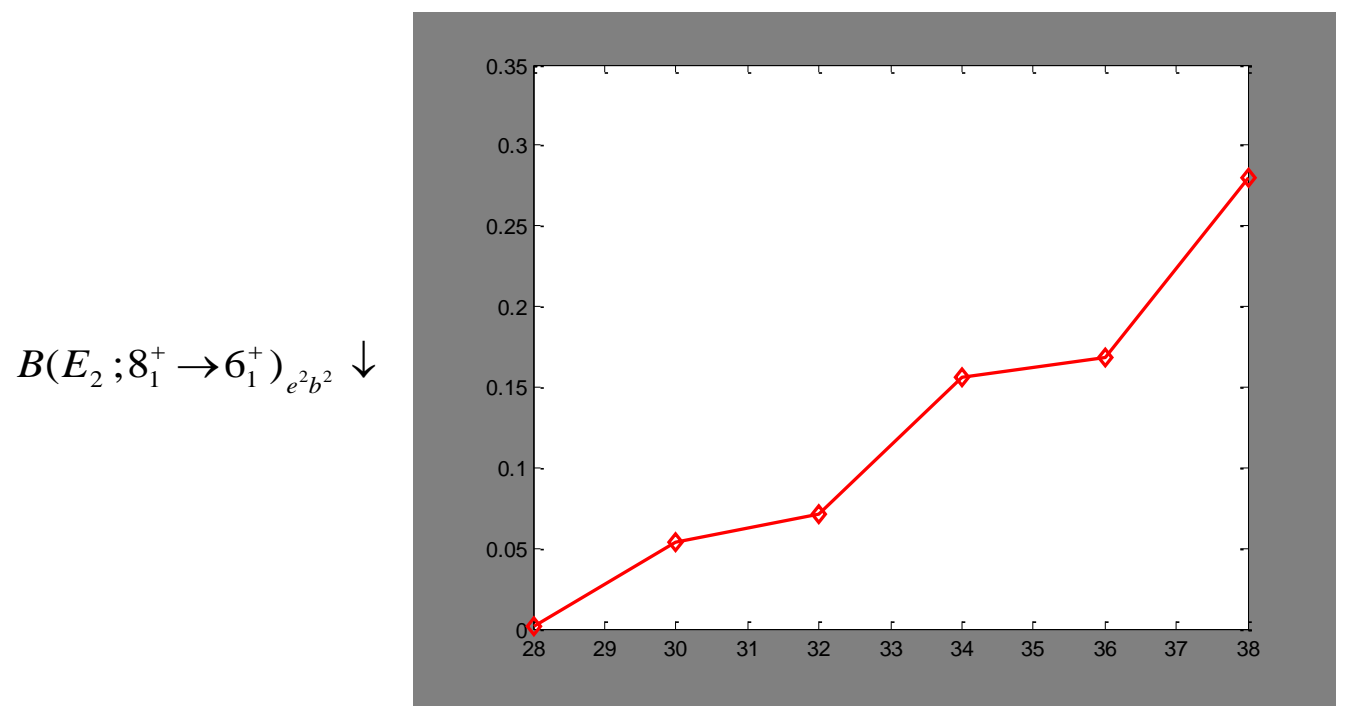

Atomic Number $(Z)$

الثكل (ץ) :- احتمالية الانتقال رباعية القطب الكهربائية المختزلة كدالة للعدد

$$
\text { الذري للنوى })
$$

ورسمت العلاقة عامل التشوه الى عامل التشوه للجسيم المنفرد م/ /

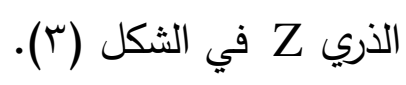

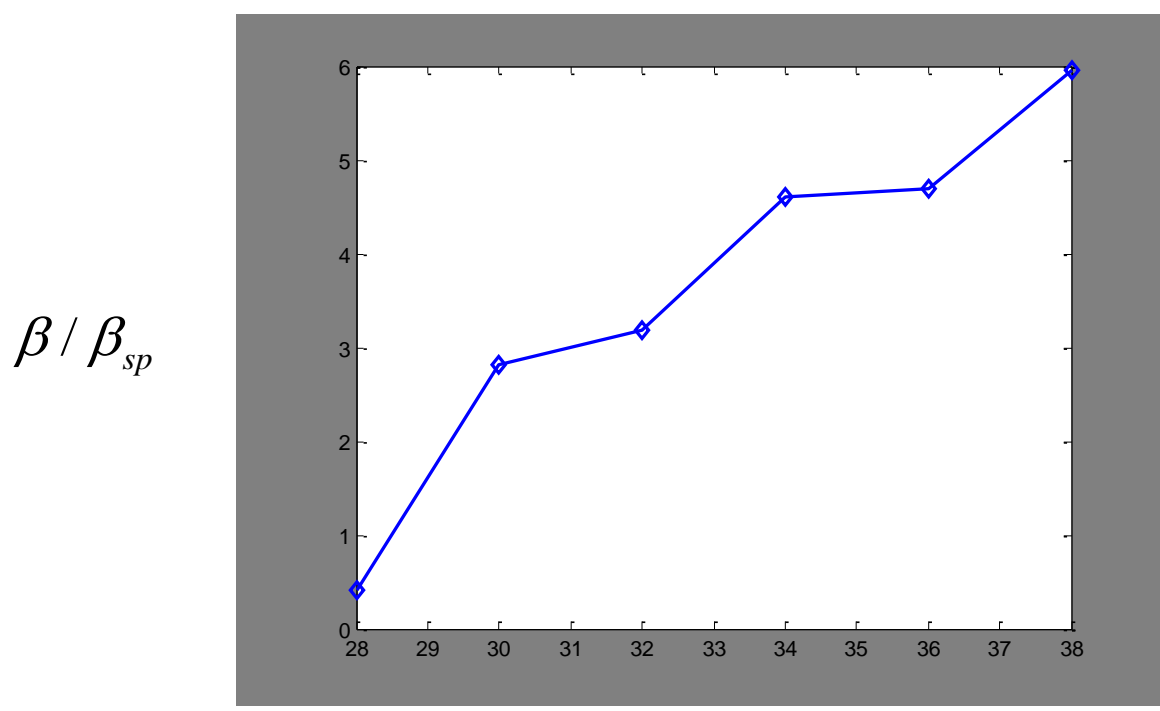

Atomic Number $(Z)$

الثكل (ץ) :- - النسبة بين عامل التشوه وعامل التشوه للجسيم المنفرد

$$
\text { الذري }
$$


تم في هذا البحث حساب طاقة الحالات الايزوميرية +8 لبعض الايزوتونات ل للنوى ( العملية المتوفرة لاحتمالية الانتقال رباعية القطب الكهربائية المختزلة النوى في حين ان هذه الاحتمالية حسبت للنواتين البوزونات المتفاعلة IBM-1 لعدم توفر قيم عملية لها.

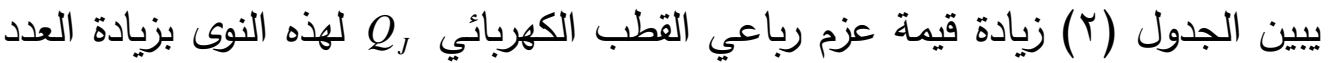

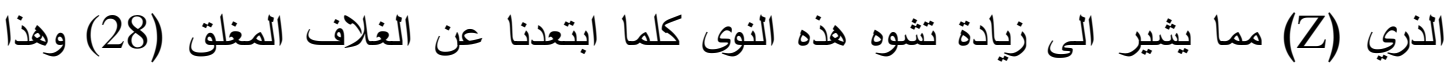
تصرف يشابه تصرف النواة في حالتها الارضية. الجدول (r) يبين نفس التصرف للنسبة B / $\beta_{2 s p}$ لها تعود للنواة Ge وهذا التصرف يخالف الحالة الارضية لهذه النوى على اعتبار ان في الحالة الارضية يزداد التشوه كلما ابتعدنا عن Z=28 وصولا الى منتصف الغلاف ثم يقل تشوها.

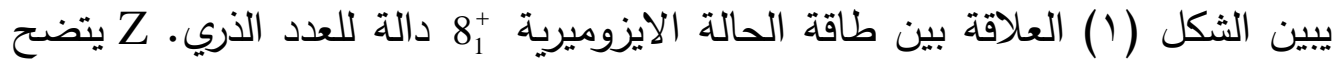

من هذا الثكل ان اقل قيمة للطاقة لهذه الحالة تعود للنواة

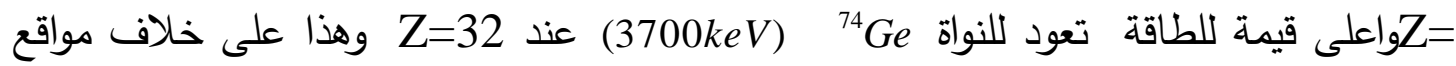

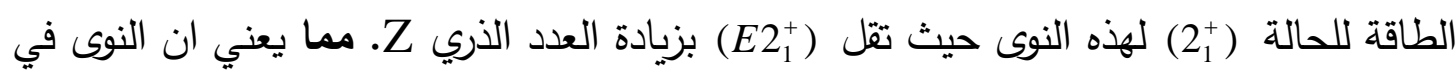

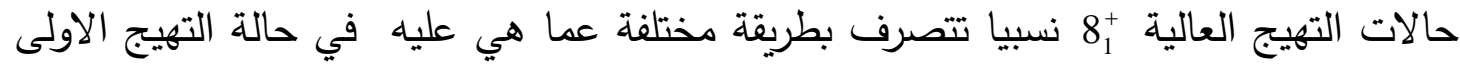
$\cdot\left(2_{1}^{+}\right)$

الشكل (r) يبين زيادة قيمة (

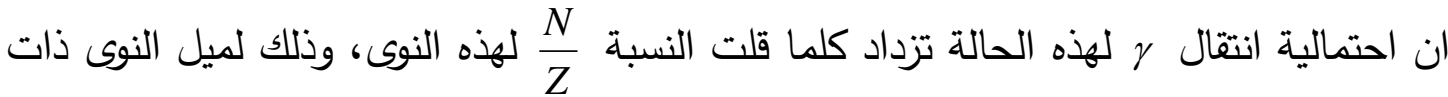
الكبيرة للانحلال بـ $\frac{N}{Z}$

حيث تقل

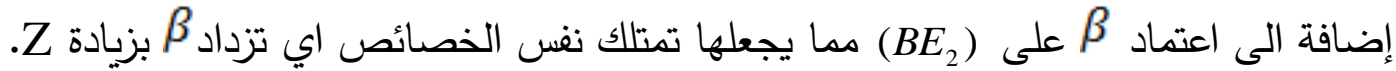

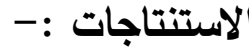

تختلف قيم مواقع الطاقات للحالة +8 لهذه النوى في تصرفها عما هو عليه للحالة (2)

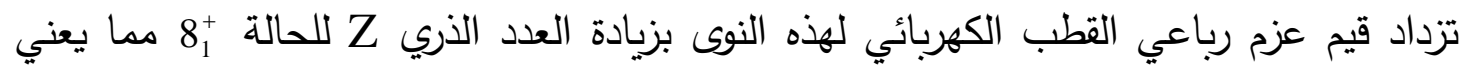

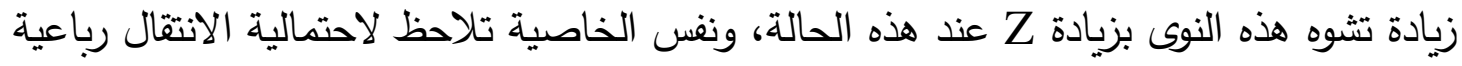




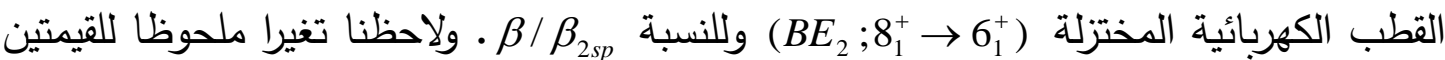

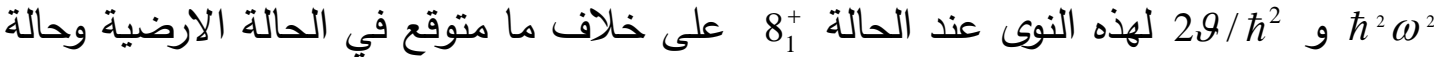

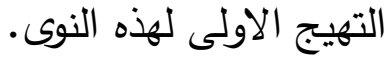

1. Abdullah,H.Y.,Hossain,I.,Ahmed,I.M.,Ahmed,S.T.,Karwan,W.Q., Kasimin, M.K., Chong, C., Viswanathan, K.K., andIbrahim, N.; (2011) "Calulation of $8_{1}^{+}$Isomers of Even-EvenNuclei ${ }^{76} \mathrm{Ni}$ to ${ }^{94} \mathrm{pd}$ forN=48Neutrons". Int.J. phy.Sc., 6(4):901-907,18.

2. Arima, A., Iachello, F., (1987) "The Interacting Boson Model (3)" CambridgeUniversity press,(51).

3. Ameenah R. Farhan, Balraj S.,(2009), Nuclear Data Sheets 110, 1917

4. Balraj S., Ameenah R. F.,(2006)"Nuclear Data Sheets "107, 1923

5. Balraj S.,(2005) "Nuclear Data Sheets" 105,223.

6. Balraj S.,(1995)" Nuclear Data Sheets" 74,63.

7. Cohen,B.L.,(1971) " Concepts Of Nuclear Physics " Megraw-Hall pub.comp.

8. Chandan(2004),CitedbyAbdullah,H.Y.,Hossain,I.,Ahmed,I.M.,Ahmed ,S.T.,Karwan,W.Q.,Kasimin,M.K.,Chong,C., Viswanathan,K.K., and Ibrahim,N.;(2011)"Calulation of $8_{1}^{+}$Isomers of EvenEvenNuclei ${ }^{76} \mathrm{Ni}$ to ${ }^{94} \mathrm{pd}$ forN=48Neutrons".Int.J.of the phy.Sc., 6(4):901-907,18.

9. Daugas,J.M.,Faul,T.,Grawe,H.,Pfützner,M.,Grzywacz,R.,Lewitowicz, M.,Achouri,N.L.,Angélique,J.C.,Baiborodin,D,,Bentida,R.,Béraud,R., Borcea,C.,Bingham,C.R.,Catford,W.N., Emsallem,A., de France,G., Grzywacz,K.L., Lemmon,R.C., Lopez Jimenez,M.J.,deOliveira Santos,F., Regan,P.H., Rykaczewski,K., Sauvestre,J.E., Sawicka,M., and Stanoiu,M.,(2010) " Low-Lying isomeric Levele in ${ }^{75} \mathrm{Cu}$ ", phys,Rev C81,034304.

10.Diriken,J., Stefanescu,I., Balabanski,D., Blasi,N., Blazhev,A., Bree,N., Cederkäll,J., Cocolios,T.E., Davinson,T.,Eberth,J., Ekström,A.,Fedorov,D.V.,Fedosseev.V.N.,Fraile,L.M., Franchoo,S., Georgiev,G., Gladnishki,K., Huyse,M., Ivanov,O.V., Ivanov, V.S.,Iwanicki,J.,Jolie,J.,Konstantinopoulos,T.;Th. Kröll., Krücken,R., Köster,U., Lagoyannis,A., Lo Bianco,G., Maierbeck, P.,Marsh,B. A.,Napiorkowski,P.,Patronis,N.,Pauwels,D., Reiter,P., Seliverstov,M., Sletten,G., Van de walle,J.,VanDuppen,P., Voulot,D., Walters, W.B.,Warr,N.,Wenander,F., and Wrzosek,K.K.,(2010) " Coulomb Excitation Of ${ }^{73} \mathrm{Ga}$ " ,Phys,Rev,C 82,064309. 
دراسة الحالة الايزوميرية $8_{1}^{+}$

11.El-Khosht,M.,(1993)"Transition Rates In Level Structure Of Doubly Even Ytterbium Isotopes " II Nuovo Cimento A,(19711996), 106(7):875-883.

12.Flanagan,K.T.,Vingerhoets,P.,Avgoulea,M.,Billowes,J.,Bissell,M.L.,B laum,K.,Cheal,B.,DeRydt,M.,Fedosseev,V.N.,Forest,D.H.,Geppert, Ch.,KÖster,U.;Kowalska,M.;Krämer,J.;Kratz,K.L.;Krieger,A.;Mané,E .;Marsh,B.A.;Materna,T.;Mathieu,L.;Molkanov,P.L.;Neugart,R;Neyen s,G.;NÖrtershäuser,W.;Seliverstov,M.D.;Serot,O.;Schug,M.;Sjoedin, M.A.;Stone,J.R.;Stone,N.J.;Stroke,H.H.;Tungate,D.;Yordanov,D.T.; Volkov,YU.M.; (2009)"Nuclear Spins and Magnetic Moments of ${ }^{71,73,75} \mathrm{C} u$ :Inversion of $\pi 2 P_{3 / 2}$ and $\pi 1 f_{5 / 2}$ Levels in ${ }^{75} \mathrm{Cu}$ " Phys, Rev, Lett, 103,142501.

13.Gorska,M.,Grawe,H.,Foltescu,D.,Fossan,D.,Grzywacz,R., Heese ,J.,Maier,K.,Rejmund,M.,Roth,H.,Schubart,R.,(1995) "Protonneutron interaction at $N=Z$ First observation of the $T z=1$ nucleus 94Pd48 inbeam". Z.Phys Hadrons Nuclei, 353(3): 233-234.

14.Grawe,H.,Langanke,K.,Martinez Pinedo,G., (2007) "Nuclear Structure and Astrophysics" Reports on progress in phys , 70:9:1525-1582.

15.Mark,A.C.,(2003)"Structure of collective model in transitional and deformed nuclei" ph.D.thesis, Yala university.

16.Raman S, Nestor Jr C, Tikkanen P (2001)" Transition Probability From The ground to First- Excited $2_{1}^{+}$State Of Even-Even Nuclides.Atomic Data Nucl.Data Tab.,78(1):1-128.

17.Raman S, Malarkey C, Milner W, Nestor C (1987). Transition Probability $\left(B E_{2}\right)$ [short up arrow],from the ground to the first excited $2_{1}^{+}$State of Even- Even Nuclides " Atomic Data Nucl.Data Tab., 36(1):1-96.

18.Sawicka,M.,Pfützner,M.,Grzywacz,R.,Daugas,J.,Matea,I.,Lewitowice, M.,Grawe,H.,Becker,F.,Belier,G.,Bingham,C., (2003) " Evidence For An Isomer In ${ }^{76} N i \quad$ ".Eur.Phys.j.A-Hadrons Nuclei,20(1):109-110.

19. Sonzogni,A.A.,Abriola,D.,(2010)"Nuclear Data Sheets " 111,1.

20.Sun,Y(2008)" Projected Shell Model description for nuclearisomers. Revista Defisca, S54(3):122-128.

21.Tuli.J. K ,(2004) "Nuclear Data Sheets" 103, 389 (2004)16-Jan.

22.Vande Walle,J.,Aksouh,F., Ames,F., Behrens,T., Bildstein,V., Blazhev, A., Cederkäll,J., Clément,E., Cocolios, T.E.,Davinson, T., Delahaye,P.,Eberth,J.,Ekström,A.,Fedorov,D.V.;Fedosseev,V.N.,Frail e,L.M.,Franchoo,S.,Gernhauser,R.,Georgiev,G.,Habs,D.,Heyde,K.,Hu ber,G.,Huyse,M.,Ibrahim,F.,Ivanov,O.,Iwanicki,J., Jolie,J., Kester,O., Köster,U., Kröll,T., Krücken,R., Lauer,M., Lisetskiy,A.F., Lutter,R., Marsh,B.A., Mayet,P., Niedermaier,O., Nilsson,T., Pantea,M., 
Perru,O., $\quad$ Raabe,R., $\quad$ Reiter,P., $\quad$ Sawicka,M., $\quad$ Scheit,H., Schrieder,G.,Schwalm,D., Seliverstov,M.D.,Siebrr,T.,Sletten. G., Smirnova,N., Stanoiu,M., Stefanescu,L.,Thomas,J.C., ValienteDobón,J.J., Van Duppen,P.,Verney,D., Voulot,D., Warr,N., Weisshaar,D., Wenander,F.,Wolf,B.H., and Zielińska,M.,(2007) "Coulomb Excitation Of Neutron-Rich Zn Isotopes : First Observation Of the $2_{1}^{+}$State in ${ }^{80} Z n$ ". Phy,Rev ,Let, 99, 142501.

23.Venkova T, Andrejtscheff W (1981). "Transition strengths B (E2) in the yrast bands of doubly even nuclei ". Atomic Data Nucl. Data Tab., 26(2): 93-136.

24. Wong, S.M.,(1990)" Introductory Nuclear Physics " Prentice-Hall International ,Inc,(268-273). 